\title{
Molecular Dynamics Study of Polymer Diffusion
}

\author{
Phillip Choi \\ Department of Chemical and Materials Engineering \\ University of Alberta
}

With ever-increasing demand on the performance of materials exhibiting unique functional properties, design of such materials and the associated manufacturing processes has become fairly sophisticated. This, in turn, requires a deeper understanding of the corresponding structure-processing-property relationships. And in some cases, it is highly desirable to have molecular level of understanding. With advances in computer technology and developments in molecular simulation methods, complex molecular systems and physical phenomena of engineering importance can be investigated with ease. Such simulation provides an effective means to determine how molecular structure and atomic-level interactions determine macroscopic properties of materials of interest as well as how they response to the imposed processing conditions. In this keynote address, I will present some basic principles and techniques used in molecular simulation and research problems in the area of polymer diffusion that my group has embarked on over the past decade using such techniques. In one example, I will demonstrate how molecular simulation help design a biopolymer that has the potential to be used for food packaging applications. 